\title{
Liver tumors and loco-regional therapy
}

\author{
Wolfgang Vogel
}

Published online: 28 February 2013

(C) Springer-Verlag Wien 2013

Hepatocellular carcinoma (HCC) is the fastest increasing malignant disease in the Western world. According to the WHO accredit Tyrolean cancer registry, the incidence in the Tyrol province of Austria has almost tripled within the last 2 decades particularly in men. Mortality, however, has only increased by 1.7 times. Incidence and mortality in the female population has almost stayed unchanged. These epidemiologic developments are probably representatives for the general situation in Austria. In addition, there is an increasing disease burden by nonmalignant liver tumors, accidentally detected by the readily-available diagnostic imaging armamentarium with increasing sensitivity such as ultrasonography, computed tomography and magnetic resonance scanners.

For years, surgical resection of the tumor was the only therapeutic option, and was considered gold standard. However, short-term outcome and long-term survival was limited in more than $90 \%$ of the patients by complications of the underlying cirrhosis. Only the development of liver transplantation in highly selected cirrhotic patients offers the option of cure for this disease with otherwise dismal prognosis. If this very expensive form of therapy is applied in an early stage of tumor disease ("Milan Criteria"), cure in the true sense of the word is achievable.

Liver tumors in cirrhotic or noncirrhotic livers usually develop for a long time without causing any clinical symptoms. As more than $90 \%$ of HCC need the environment of the chronically damaged organ, surveillance of patients at risk improves detection rate at an early stage. But still, there remains a large group of patients with either tumor stage outside the transplant criteria or other contraindications to transplantation. Furthermore, this expensive form of treatment is not generally available.

Univ.-Prof. Dr. W. Vogel $(\bowtie)$

Universitätsklinik für Innere Medizin II, Gastroenterologie \& Hepatologie, Department Innere Medizin, Medizinische Universität Innsbruck, Anichstraße 35, 6020 Innsbruck, Austria e-mail: wolfgang.vogel@i-med.ac.at
There are two further milestones in the therapy of malignant liver tumors: the introduction of the first effective medical therapy, the multikinase inhibitor sorafenib, and the introduction of loco-ablative treatment modalities.

Stage-oriented management of HCC is based on the stage of the cirrhosis, usually expressed as Child-Pugh stage $\mathrm{A}-\mathrm{C}$, the performance state of the patient, and size and number of tumor nodules. Curative liver transplantation is considered for tumors less than three of maximum $3 \mathrm{~cm}$ in size, or a single tumor of maximum $5 \mathrm{~cm}$ in size. Surgical resection and loco-regional therapies are palliative in its true meaning as HCC will, at some time, recur somewhere else in the cirrhotic liver. Medical treatment with sorafenib will stop further tumor growth. Surgical resection can offer a 5-year-survival of $70 \%$ for an adequately selected patient with a single tumor smaller than $5 \mathrm{~cm}$-either de novo or metastatic-in the liver of $70 \%$ at 5 years. The role of loco-ablative therapy of small tumors $<2 \mathrm{~cm}$ as a competitive first-line alternative to resection remains controversial, as no randomized controlled trial has answered this question with confidence.

Percutaneous ethanol injection (PEI) of liver tumors was experimentally pioneered in the early eighties of the last century and for its safety and efficacy, was soon introduced into clinical practice. It appeals for its simple and cheap way of application with ultrasonography guidance and its reasonable effectiveness. After the proof of principle, more sophisticated ways of local tumor destruction were developed as transcatheter arterial chemo (radio)embolization (TAC(R)E), radiofrequency ablation (RFA), and other still experimental methods.

Recommendations for loco-regional therapies are based on evidence from a recent metaanalysis of pooled data and these are as part of the concept of HCC treatment endorsed by the major international hepatologic and oncologic societies. Local therapies for HCC are standard for patients not suitable for surgery and achieve 5-year survival rates of 50-70\%. In addition, this modality can be offered to patients with rare benign liver tumors that need treatment as an effective alternative to surgery, 


\section{editorial}

when an operation is not possible. RFA is the first option for single tumors and the second option is PEI, if RFA is not possible or available. In patients with multinodular disease, TACE can delay progression and prolong survival beyond $30-40$ months provided there is no extrahepatic spread and preserved liver function.

In this special issue of Wiener Medizinische Wochenschrift, leading experts from Medical University Inns- bruck will update revue clinical, diagnostic, and loco-regional therapeutic aspects. This tertiary Austrian center has a long-standing internationally recognized reputation in the treatment of liver disease. The department of radiology is particularly known for its pioneering work in computed tomography assisted RFA of HCC and cholangio-cellular carcinoma. 